\title{
Bildgebende Diagnostik bei Verletzungen des Fußes
}

\author{
Matthias Beilicke, Rainer Braunschweig
}

\section{Zusammenfassung}

Die Verfahren der modernen bildgebenden Diagnostik sind vielfältig. Zur Verfügung stehen unterschiedliche technische Grundprinzipien. Hieraus ergeben sich Indikationen und Leistungsspektren. Der Nachweis oder Ausschluss ossärer Verletzungen wird primär mit der Projektionsradiographie in 2 Ebenen geführt. Diese Basisdiagnostik wird ergänzt durch gehaltene Aufnahmen. Im Rahmen einer erweiterten Basisdiagnostik kommt die CT als überlagerungsfreies Schnittbildverfahren zum Einsatz. Im Vordergrund stehen operationstaktische Fragen.
Die Kernspintomographie ist eine Methode der Spezialdiagnostik, die frühzeitig (postprimär) bei okkulten Knochen- und Weichteilbefunden und zur Erstellung eines Ausgangsbefundes für die spätere Begutachtung ihre Berechtigung hat. Sekundär kann die MR bei Verlaufskontrollen, insbesondere bei intra- und parossären Komplikationen, therapierelevante Zusatzinformationen liefern. Im Rahmen der Begutachtung kommt der MR ein wesentliches Leistungsspektrum zu. Der oft schwierige Fragenkomplex nach Befundobjektivierung und -differenzierung einerseits und der Kausalität andererseits ist hochsensitiv und spezifisch zu beantworten.

\section{Einleitung}

Bei Verletzungen des Fußes steht neben der Anamnese und klinischen Untersuchung vor allem die bildgebende Diagnostik im Vordergrund. Folgende Fragen sind zu beantworten:

1. Befundnachweis oder -ausschluss

2. Begleitbefunde

3. operationstaktische Überlegungen

4. Verlaufs-/Stellungskontrolle; Frakturheilung

5. Nachweis bzw. Ausschluss von Komplikationen

6. Begutachtung.

Die moderne bildgebende Diagnostik setzt bei Verletzungen des Fußes unverändert die Projektionsradiographie (syn.: Röntgen in 2 Ebenen) als Methode der ersten Wahl ein. Daneben sind als Schnittbildverfahren die Computertomographie, die Kernspintomographie und

OP-JOURNAL 2001; 17: 14-18

(c) Georg Thieme Verlag Stuttgart · New York

Sonographie etabliert. Technische Grundlagen der Bildgebung, Vor- und Nachteile, insbesondere die wesentlichen Leistungsspektren der genannten Methoden sind in Tab. 1 aufgelistet.

Zur traumatologischen Diagnostik stehen verschiedene bildgebende Verfahren zur Verfügung. Ihre Indikationen, Leistungsspektren sowie ihre Aussagefähigkeit ergeben sich aus den zugrundeliegenden technisch-physikalischen Prinzipien.

\section{Erstdiagnostik}

Bei Neuaufnahme eines Patienten steht im Rahmen der Erstdiagnostik der Nachweis oder Ausschluss ossärer und/oder Weichteilverletzungen im Vordergrund. Die erforderliche bildgebende Basisdiagnostik (s.u.) ist die standardisierte Röntgenaufnahme in 2 Ebenen [10,11].

Die technischen Entwicklungen in den letzten Jahren haben hierfür digitale projektionsradiographische Techniken für den Routineeinsatz zur Verfügung gestellt. Vorteil dieser Methode ist die hohe konstante Bildqualität und die Möglichkeit, in angeschlossene digitale $\mathrm{Ar}$ chivmedien und vor allem Bildverteilungsnetze (Notaufnahmen, Intensivstationen, OP-Säle, Stationen) innerhalb eines Krankenhauses einzuspeisen. In modernen Traumazentren sind die Bilder sofort und an mehreren Orten verfügbar. Zeitaufwendige Entwicklungs- und $\mathrm{Ar}$ chivierungsvorgänge und zeitlich aufeinanderfolgende Bildanalysen unterschiedlicher klinischer Bereiche (Chirurgie, Anästhesie, Innere Medizin etc.) sind entbehrlich. Die Entscheidungsprozesse verkürzen sich im Interesse einer schnellen Erstversorgung erheblich [8].

Zur bildgebenden Darstellung der komplexen Architektur bzw. Anatomie des Fußes, den wir aus funktionellen und didaktischen Gründen in das obere und untere Sprunggelenk, den Rückfuß (Kalkaneus) sowie den Mittel- (Tarsalia und Metatarsalia) und Vorfuß (Phalangen) einteilen, stehen zusätzlich projektionsradiographische Spezialeinstellungen zur Verfügung, die Einzelfragestellungen z.B. nach Kalkaneusfrakturen, Beteiligung des unteren Sprunggelenkes bei Talusfrakturen, Luxationsfrakturen des Lisfrancschen Gelenkes oder Fissuren bzw. okkulte Frakturen an Einzelknochen des Fußskeletts (z. B. Basisfraktur des Os metatarsale V oder Navicularfrakturen) beantworten helfen (Abb.1). Dem Thema entsprechend, werden wir auf klinische Klassifikationen und Therapieansätze in unserer Darstellung nachfolgend verzichten. Typische Fraktursituationen sind in Abb. 2 und $\mathbf{3}$ dargestellt.

Bei projektionsradiographisch ausgeschlossenem Frakturverdacht können im dritten Schritt bei entsprechendem klinischen Befund und Verletzungsmechanismus „gehaltene Aufnahmen“ (Basisdiagnostik) erstellt werden. Ziel ist, den ligamentären Stabilisierungsapparat der Gelenke in Provokationsstellung zu 
Tab. 1 Charakteristika bildgebender Verfahren

\begin{tabular}{|c|c|c|c|c|}
\hline Verfahren & Charakteristik & Vorteile & Nachteile & Besonderheiten \\
\hline Röntgen & ionisierende Strahlung & $\begin{array}{l}\text { - schnell, billig } \\
\text { - flächendeckend verfügbar } \\
\text { - reproduzierbar }\end{array}$ & $\begin{array}{l}\text { - Überlagerungen } \\
\text { - Strahlenexposition }\end{array}$ & $\begin{array}{l}\text { - hohe Detailerkennbarkeit } \\
\text { - konventionelle Tomogra- } \\
\text { phie }\end{array}$ \\
\hline $\mathrm{CT}$ & ionisierende Strahlung & $\begin{array}{l}\text { - überlagerungsfreie } \\
\text { Darstellung } \\
\text { - 3D-Rekonstruktionen }\end{array}$ & - Strahlenbelastung & $\begin{array}{l}\text { - hoher Qualitätsstandard } \\
\text { - dritte Ebene }\end{array}$ \\
\hline MRT & elektromagnetische Wellen & $\begin{array}{l}\text { - hervorragender Weichteil- } \\
\text { kontrast } \\
\text { - keine Schädigungen } \\
\text { bekannt }\end{array}$ & $\begin{array}{l}\text { - störanfällig } \\
\text { - Cave: z.B. Metallimplantate }\end{array}$ & $\begin{array}{l}\text { - hohe Sensitivität } \\
\text { - teilw. hohe Spezifität }\end{array}$ \\
\hline Sonographie & $\begin{array}{l}\text { Darstellung von Impedanz- } \\
\text { unterschieden }\end{array}$ & $\begin{array}{l}\text { - schnell } \\
\text { - kostengünstig }\end{array}$ & $\begin{array}{l}\text { - untersucherabhängig } \\
\text { - schlecht reproduzierbar }\end{array}$ & $\begin{array}{l}\text { - Weichteilbefunde } \\
\text { (z.B. Sehnen) } \\
\text { - beliebige Schnittorien- } \\
\text { tierung }\end{array}$ \\
\hline
\end{tabular}

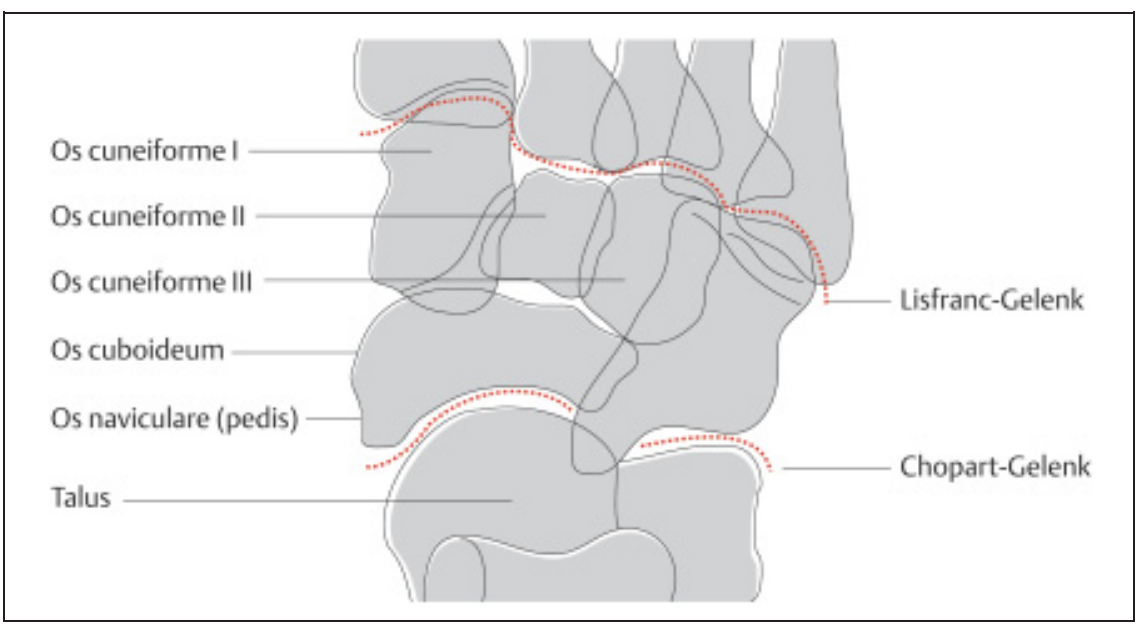

Abb.1 Schematische Darstellung der Fußwurzelgelenke nach Chopart und Lisfranc (Abb. entnommen aus 2).

bringen und ggf. durch eine vermehrte Gelenkaufklappbarkeit eine Verletzung der Bandstrukturen nachzuweisen oder auszuschließen. Hierzu existiert eine Vielzahl messtechnisch erfassbarer Parameter (Winkelmaße, Knochenvorschub etc.) [6].

Wir setzen „gehaltene Aufnahmen“ mehrheitlich für das obere Sprunggelenk (Abb.4) und das Talonavikular- sowie Kalkaneocuboidalgelenk ein.

Der primäre Nachweis oder Ausschluss von Frakturen sowie die Heilungskontrolle bei nachgewiesener Fraktur erfolgt durch die Projektionsradiographie in 2 Ebenen als bildgebende Basismethode. "Gehaltene Aufnahmen" kommen ergänzend zum Einsatz.

Im Gegensatz zur Projektionsradiographie, die einen dreidimensionalen Körper in einer zweidimensionalen Fläche abbil- det und eine exzellente Detailerkennbarkeit der Hochkontraststrukturen erlaubt, wird die Computertomographie (CT) als röntgendiagnostisches Verfahren zur Erfassung der dritten Ebene (axiale Schichten) und überlagerungsfreien Darstellung von ossären und Weichteilbefunden eingesetzt. Wir verstehen die CT als erweiterte Basisdiagnostik (s.u.).

Neben dem Nachweis etwaiger okkulter Frakturen (z.B. Kalkaneusfrakturen, Talusimpressionen - flake fractures etc.) ist die Domäne der Computertomographie vorrangig die Beantwortung operationstaktischer Fragen bei der Planung des operativen Vorgehens. Dies trifft insbesondere für den Kalkaneus, die etwaige Mitbeteiligung von Gelenken (z.B. unteres Sprunggelenk) und die damit erforderliche Quantifizierung von Gelenkstufen und/oder gelenktragender Fragmente, aber auch z. B. bei komplexen Verletzungen des Mittelfußes zu (Abb.5 u.6).

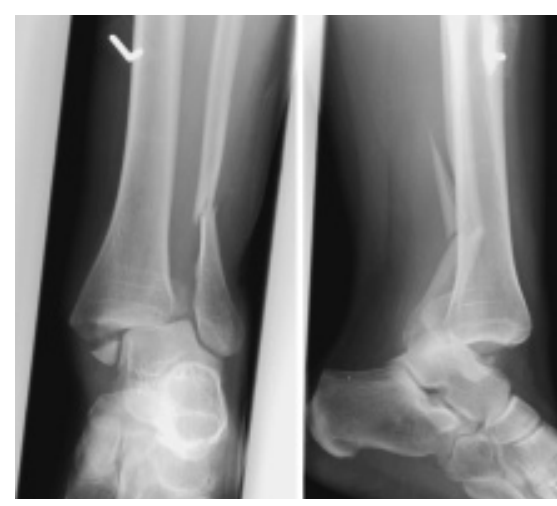

Abb.2 Trimalleoläre Luxationsfraktur des oberen Sprunggelenkes.

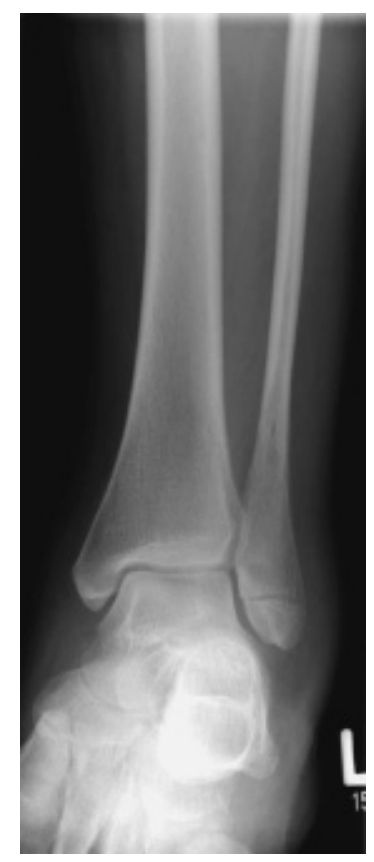

Abb. 3 Weber-A-Fraktur des lateralen Malleolus. 


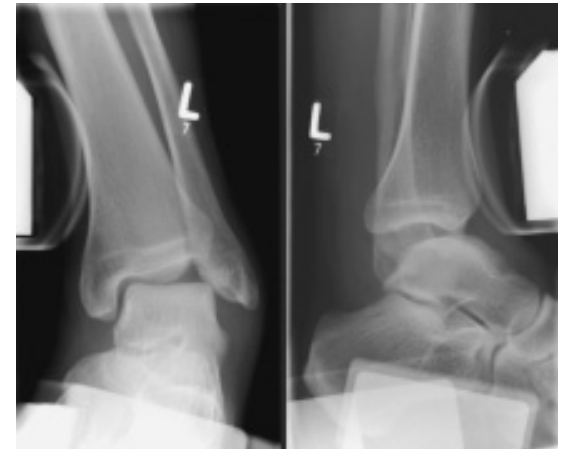

Abb.4 Gehaltene Aufnahmen des oberen Sprunggelenkes mit verstärkter „Aufklappbarkeit" des Gelenkspaltes. Radiologische Zeichen einer Bandläsion.
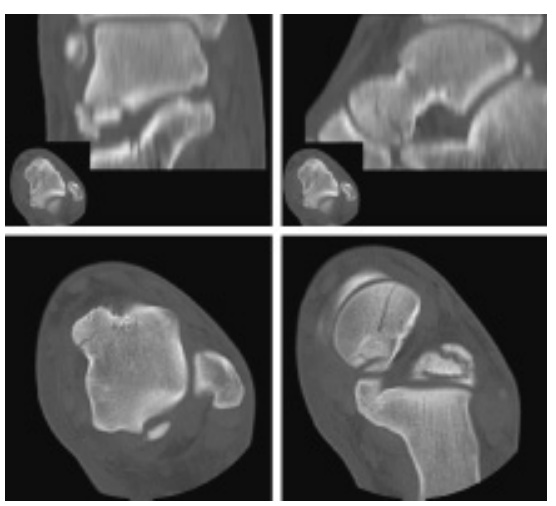

Abb.5 Mehrfragmentäre Talusfraktur. Die multilokuläre Fraktursituation wird neben den originären Schnittbildern besonders durch die Sekundärrekonstruktionen verdeutlicht.

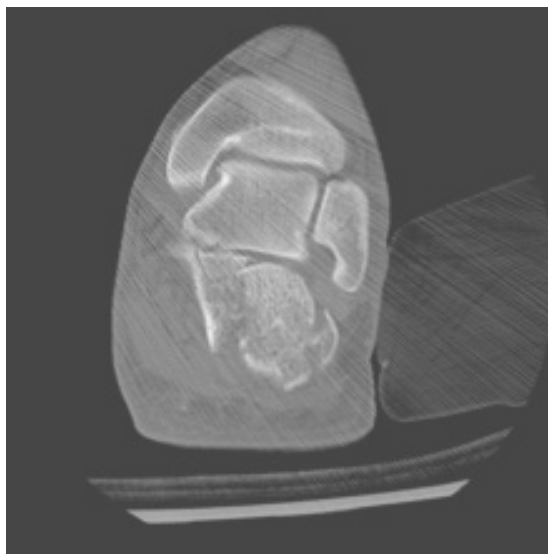

Abb.6 Mehrfragmentäre Kalkaneusfraktur mit Beteiligung der subtalaren Gelenkfläche.

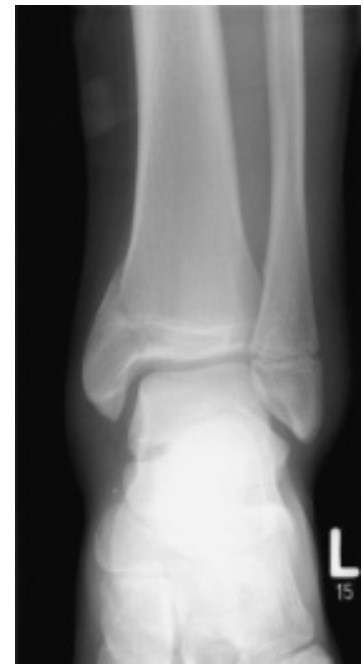

Abb. 7 Aitken-3-Fraktur eines 15-jährigen Jugendlichen.

Die Computertomographie dient überwiegend der Klärung operationstaktischer Fragestellungen und der Verfizierung von Frakturheilungsstörungen.

Die Kernspintomographie (MR), die auf der Basis elektromagnetischer Hochfrequenzwellen arbeitet, wird wesentlich charakterisiert durch ihren exzellenten Weichteilkontrast. Hieraus ergeben sich ihre Indikationen. Die Kernspintomographie steht im Rahmen der Spezialdiagnostik (s.u.) zur Verfügung. Perakute Fragestellungen ergeben sich im Bereich des Fußes nicht!

Veränderungen des gesamten Weichteilmantels sind darstellbar. $\mathrm{Zu}$ erwähnen sind ligamentäre, muskuläre und in Einzelfällen auch neuronale Strukturen. Hinzu kommen Darstellungsmöglichkeiten ossärer Befunde. Die ossären Strukturen selbst sind kernspintomographisch infolge des weitgehenden Fehlens der für die MR wesentlichen Wasserstoffprotonen-

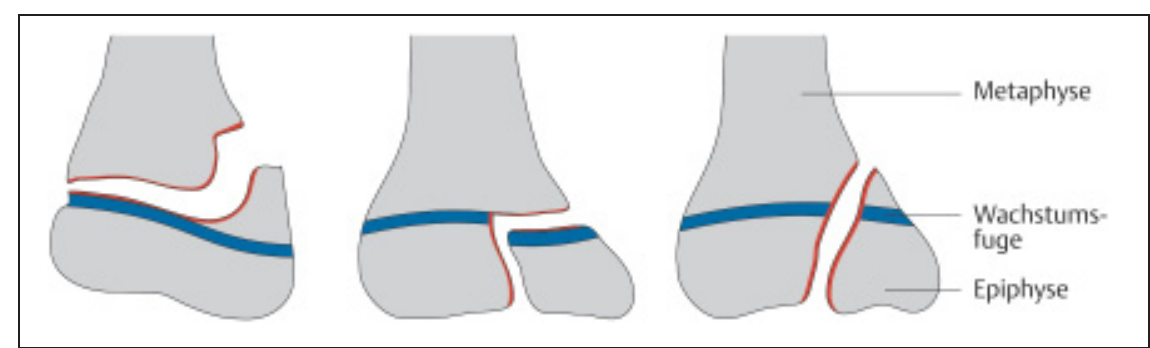

Abb. 8 Einteilung der kindlichen Sprunggelenksfrakturen mit Beteiligung der Wachstumsfuge nach Aitken (Abb. modifiziert aus 2). konzentration nicht hinreichend fassbar. Die „Knochendarstellung“ erfolgt über die Signalgebung des Knochenmarks bzw. seiner im pathologischen Fall entsprechend veränderten kernspintomographischen Signalintensitäten. Dies bezieht sich auf Wassereinlagerung (Wasserstoffprotonenkonzentration erhöht $=$ Ödem), auf Einblutungen oder Entzündungen (Blutabbauprodukte, Zellreichtum, Ödem) oder tumoröse Veränderungen (Ödemzonen, Destruktionen, Markkonversionen) extra- bzw. intraossär. Im Rahmen von Fußverletzungen klinisch relevant sind zudem okkulte Frakturen (s.o.) oder ossäre Mikrotraumatisierungen (z.B. Ermüdungsbrüche) mit klinisch positivem jedoch projektionsradiographisch negativem Befund.

Bezogen auf den gesamten Fuß kommt der Kernspintomographie bei Verletzungen des oberen und unteren Sprunggelenkes eine wesentliche Bedeutung zu. Dies resultiert aus der Summe hoch vulnerabler funktionswichtiger Strukturen und ihrer Verletzungshäufigkeit.

Im Bereich des oberen Sprunggelenkes sind z.B. Läsionen des anterioren und posterioren fibulotalaren, des fibulokalkanearen sowie des Deltabandes (axiale und koronare Schnittführung empfohlen) detektierbar. Differenzierungen sind sowohl hinsichtlich des Vorliegens einer partiellen oder kompletten Läsion als auch in Einzelfällen bezüglich des Zeitpunktes der Verletzung möglich $[3,4]$.

Im unteren Sprunggelenk können Verletzungen der Ligamente im Sinus tarsi ( $\mathrm{Li}$ gamentum talocalcaneum interosseum, Ligamentum cervicale) sowie des Kalkaneocuboidal- und des Talonavikulargelenkes erfasst werden $[3,9]$.

Eine Sonderstellung im Rahmen der Frakturdiagnostik nimmt die Kernspintomographie bei kindlichen Malleolarfraktu- 


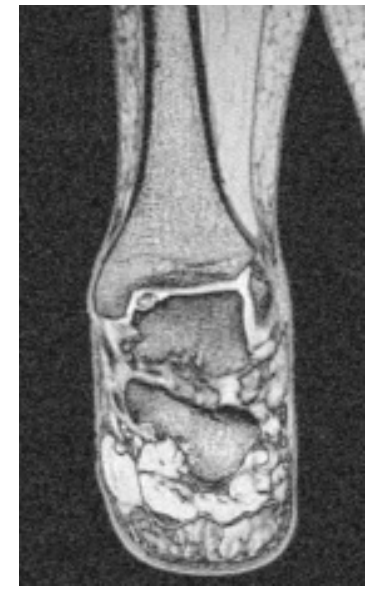

Abb.9 Kernspintomographie: Osteochondrosis dissecans der medialen Talusschulter.

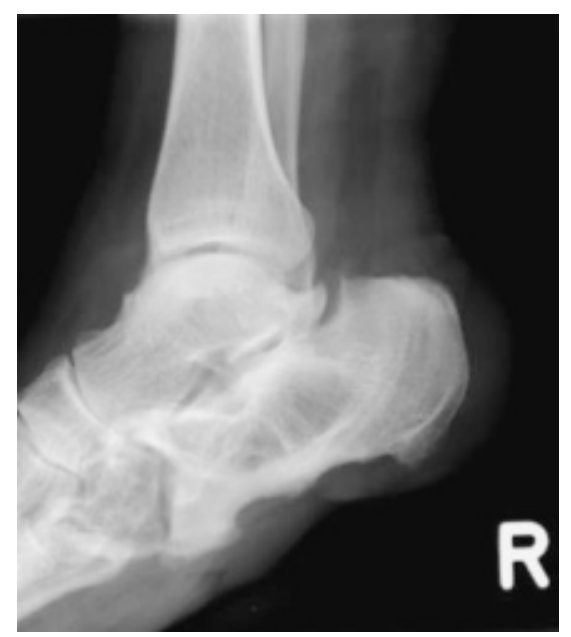

Abb.10 Komplizierte Frakturheilung 1: Chronische Osteomyelitis bei plantarem Weichteildefekt nach Kalkaneusfraktur.

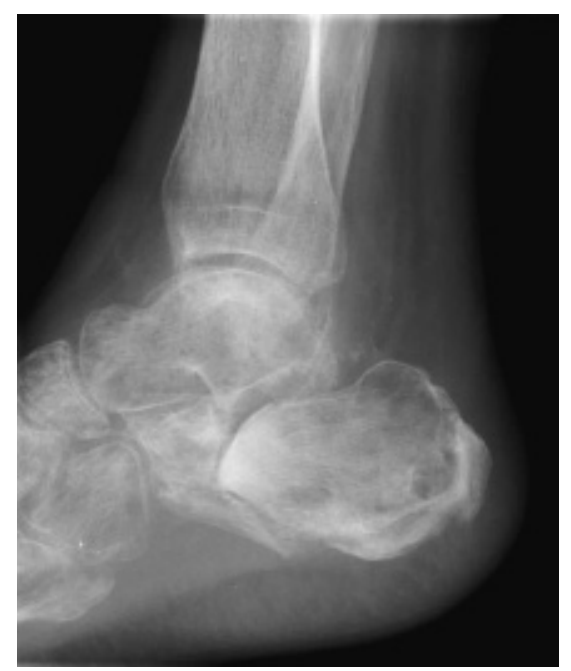

Abb.11 Komplizierte Frakturheilung 2: Kalkaneuspseudarthrose. ren mit Beteiligung der Wachstumsfugen (Klassifikationen nach Salter-Harris bzw. Aitken, siehe Abb. 7 u. 8) ein, deren Ausmaß im Projektionsbild oft unterschätzt wird. Die durch multiplanare Darstellung in der MR gewonnenen Zusatzinformationen ermöglichen eine sicherere Frakturklassifikation, woraus ein Therapiewechsel resultieren kann [7]. Im Hinblick auf die fehlende Strahlenbelastung sollte die Indikation zur kernspintomographischen Ergänzungsuntersuchung in diesen Fällen weit gestellt werden.

Okkulte und osteochondrale Frakturen stellen ein häufiges Verletzungsmuster des Talus dar. Besonders ist die Osteochondrosis dissecans der medialen Talusschulter (Abb.9) zu erwähnen, deren Verifizierung und Stadieneinteilung ebenfalls eine Domäne der Kernspintomographie ist.

Die Kernspintomographie als Methode der Spezialdiagnostik wird zur Suche nach okkulten Frakturen und Weichteilverletzungen sowie zur Erstellung eines Ausgangsbefundes für die spätere Begutachtung eingesetzt. Weiterhin liefert sie therapierelevante Zusatzinformationen insbesondere bei intra- und parossären Komplikationen.

\section{Verlaufskontrolle}

Die Frakturheilung wird routinemäßig mittels der Projektionsradiographie in 2 Ebenen dokumentiert, wozu prinzipiell Aufnahmen in den gleichen standardisierten Ebenen wie zur Erstdiagnostik angefertigt werden. Aus Gründen der Strahlenhygiene können bei komplikationslosem Heilungsverlauf im Einzelfall auch Untersuchungen auf eine Ebene beschränkt werden. Dies trifft vor allem auf Zwischenkontrollen zu. Die Abschlusskontrollen sind in 2 Ebenen $\mathrm{zu}$ erstellen.

Die Dokumentation des operativen Sanierungsergebnisses komplizierter Frakturen, z.B. von Kalkaneusfrakturen, erfolgt zweckmäßig mit der Computertomographie, die Aussagen zu Material- und Fragmentlage, Gelenkflächenverhältnissen und Konsolidierungszustand (auch von eingebrachter Spongiosa oder Endobon) ermöglicht. Multiplanare Rekonstruktionen erlauben zudem die Visualisierung in drei Ebenen. Es ist lediglich ein primärer (axialer oder koronarer - auf dem Untersuchungstisch des CT aufgestellter Fuß) Datensatz notwendig. 3D-Oberflächenrekonstruktionen ermöglichen eine zusätzliche Darstellung, sind jedoch aufgrund ihres nur unwesentlichen Informationszuwachses oft entbehrlich.

Ergeben sich klinisch Hinweise auf eine verzögerte oder komplizierte Frakturheilung (Abb.10 u.11), sollte eine großzügige Indikation $\mathrm{zu}$ weiterer bildgebender Diagnostik gestellt werden, wobei sich die einzelnen Verfahren zur Sicherung folgender Befunde eignen:

- Projektionsradiographie: Materialbruch, Fragmentdislokation, Entzündung, Dystrophie

- CT: fehlerhafte Materiallage, Arthrose, Sequestration/Abszesse

- MRT: okkulte Entzündung, Osteonekrose, M. Sudeck, ligamentäre, muskuläre, neuronale Begleitverletzungen.

\section{Begutachtung}

Mit Beendigung der Therapie und nach erfolgter Dokumentation des Heilungserfolges ist aus therapeutischer Sicht eine weitere bildgebende Diagnostik nur im Rahmen der Langzeitkontrolle (z. B. etwaige BGSW-Verfahren) und der Begutachtung erforderlich. Da lt. einem Urteil des 2.Senats des Bundesgerichtshofes die Anwendung ionisierender Strahlung ohne therapeutische Konsequenz den Tatbestand der Körperverletzung erfüllt [1], sind hier besonders strenge Maßstäbe anzusetzen. Eine Ausnahme bilden alle Fragestellungen im Zusammenhang mit der Begutachtung, bei der die soziale Indikation vor allem im Interesse des Patienten steht.

Im Rahmen berufsgenossenschaftlicher oder anderer Zusammenhangsgutachten wird ein radiologisches Fachgutachten erstellt, das folgende Fragen beantworten muss [5]:

1. Heilungszustand des Verletzungsmusters

2. Schweregrad der Verletzungsfolgen (z.B. auch inklusive umgebender Gelenkstrukturen)

3. Ursachenzusammenhang

Diese Aussagen sind überwiegend mittels Standard-Projektionsradiographie in 2 Ebenen zu treffen, wobei Voraufnahmen der Erstdiagnostik und Verlaufskontrollen in die Beurteilung einbezogen werden sollten.

Zur Differenzierung zwischen posttraumatischen und vorbestehend-degenerativen Veränderungen können im Einzelfall Vergleichsaufnahmen der (gesunden) 


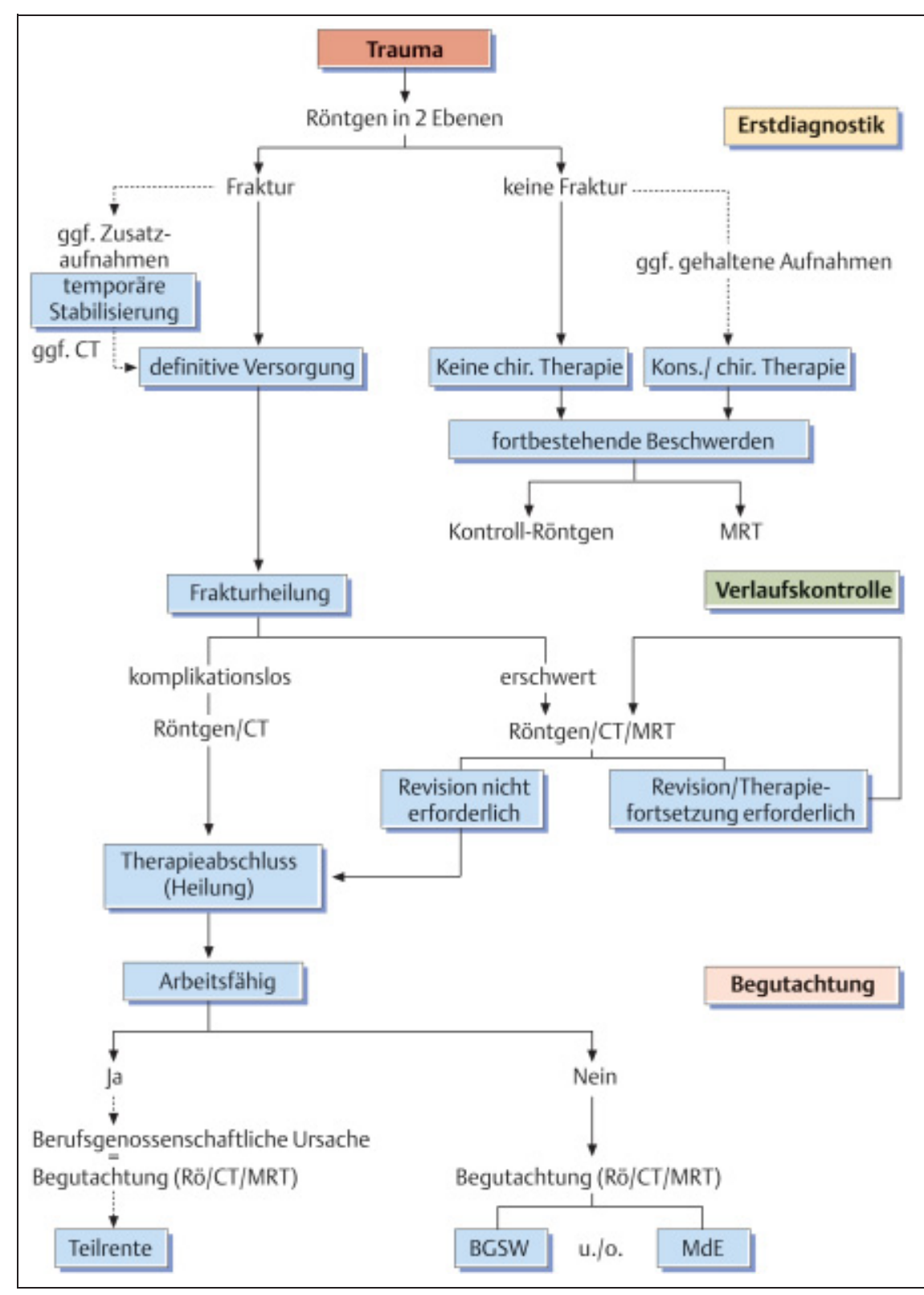

Abb.12 Schematische Übersicht des Einsatzes bildgebender Verfahren in verschiedenen Stadien der Frakturdiagnostik.

Gegenseite angefertigt werden. Diese Vorgehensweise ist jedoch aus Gründen der Strahlenhygiene streng zu indizieren. Der Fachradiologe ist in der Mehrzahl der Fälle in der Lage die o.g. Fragen gutachterlich aus dem Bildmaterial der durch den Unfall betroffenen Körperregion zu beantworten. Ein pauschales „Mitröntgen der Gegenseite“ (z. B. Hände) ist abzulehnen. Die Fachdiskussion hierzu ist nicht abgeschlossen.

Liegt ein komplexes Verletzungsmuster mit Beteiligung von Knochen und Weichteilen vor, sind ggf. Frakturheilungsstörungen aufgetreten oder ist die zu begutachtende Körperregion infolge ihrer ana- tomischen Komplexität projektionsradiographisch nicht eindeutig darstellbar (z.B. Schulter- und Kniegelenk, Fuß, Kopf und Wirbelsäule, Rückenmarksstrukturen etc.) ist die Anfertigung eines CT bzw. MR im Rahmen des Gutachtens angezeigt [5]. Die hiermit verbundenen Mehrkosten sind angesichts einer ggf. resultierenden OP-Indikation mit nachfolgender Beschwerdelinderung und zügigerer Wiedereingliederung in das Arbeitsleben zu vernachlässigen.

Besonders hinweisen möchten wir in diesem Zusammenhang auf die Verflechtung der Erstdiagnostik mit Fragen der Begutachtung. Wie bereits ausgeführt, sollten vor allem Schnittbildverfahren bei okkulten Verletzungsmustern (auffällige Klinik, „leeres Röntgenbild“) unmittelbar postprimär angefertigt werden (z.B. Schleudertrauma der HWS - Rückenmarkkontusion und/oder Vertebralisdissektion), um für die Folgebeurteilung eine Trennung zwischen Verletzungsmuster und Vorschäden dokumentieren zu können. Die bekannten langjährigen und teilweise kostenintensiven Begutachtungsverfahren können im Interesse des Patienten und der Kostenträger wesentlich abgekürzt werden.

Die Einbindung bildgebender Verfahren in die verschiedenen Stufen der Frakturdiagnostik und Verlaufskontrolle wird in Abb.12 zusammenfassend dargestellt.

\section{Literatur}

${ }^{1}$ BGH in: Neue Juristische Wochenschrift 1998; S. 833 (Urteil vom 03.12.1997, Aktenzeichen: 2 StR 397/97)

2 Bittner RC, Roßdeutscher R. Leitfaden Radiologie. Gustav-Fischer-Verlag, Stuttgart, Jena, New York, 1996

${ }^{3}$ Breitenseher MJ. (1) Akutes Trauma des Sprunggelenkes. Radiologe 1999; 39: 16-24

${ }^{4}$ Breitenseher M, (2) Trattnig S, Kukla C, Gäbler C, Helbich T, Haller J, Imhof H. Trauma von Bandapparat und Sehnen: Untersuchungstechnik und Nachweis in der MRT. Radiologe 1995; 35: 456-462

${ }^{5}$ Heuck FHW, Frik W, Scherz H-W (Hrsg.). Radiologische Fachgutachten. Springer Verlag, Berlin, Heidelberg, New York, 1999

${ }^{6}$ Heuck FHW, Bast BRG. Radiologische Skizzen und Tabellen - Peripheres Skelett. Georg Thieme Verlag, Stuttgart, New York, 1994

${ }^{7}$ Iwinska-Zelder J, Schmidt S, Ishaque N, Hoppe M, Schmitt J, Klose KJ, Gotzen L. Epiphysenfugenverletzungen der distalen Tibia. Sinnvolle Mehrinformation durch die MRT? Radiologe 1999; 39: 25-29

${ }^{8}$ Kaden I, Schwarzer J, Sprengel C, Tolksdorf, R. Braunschweig. RIS/PACS: Cost effectiveness. Vortrag auf dem European Congress of Radiology, Wien, März 2001

${ }^{9}$ Klein MA, Spreitzer MR. Imaging of the tarsal sinus normal anatomy, pathologic findings, and features of the sinus tarsi syndrome. Radiology 1993; 186: 233-240

${ }^{10}$ Lissner JU, Fink (Hrsg.). Radiologie II Enke Verlag, Stuttgart, 1990

${ }^{11}$ Meschan I. Diagnostik mit bildgebenden Verfahren. Band II: Knochen und Gelenke. Enke Verlag, Stuttgart, 1988

\section{Dr. med. Matthias Beilicke}

Facharzt f. Diagnostische Radiologie

Dr. med. Rainer Braunschweig

Facharzt f. Radiologie

Direktor der Klinik

BG-Klinik Bergmannstrost

Klinik für bildgebende Diagnostik und Interventionsradiologie

Merseburger Str. 165

06112 Halle/S. 